\title{
INGENIERÍA COLABORATIVA Y MODOS DE AUTOAPRENDIZAJE: LA EXPERIENCIA DE AULABIERTA EN LA UNIVERSIDAD DE GRANADA
}

\author{
Antonio Collados Alcaide \\ Universidad de Granada. Dpto. Escultura
}

\section{Resumen}

El artículo se centra en los modos de trabajo discente dentro de la experiencia Aulabierta (Universidad de Granada, 2004 a 2012). Aulabierta fue una comunidad experimental de aprendizaje codiseñada por estudiantes de diversas disciplinas con la ayuda de un amplio número de colaboradores, un lugar conectivo que trata de seguir la inercia de otros proyectos culturales críticos que tienen como objetivo transformar y contaminar las categorías estancas instauradas desde la modernidad en las instituciones del saber. Aulabierta trata de instituir formas disidentes de trabajar desde dentro de la academia para avanzar en la producción colectiva de conocimiento, retomando el carácter político de este como algo que es colectivamente construido.

\section{Palabras claves: AUTOFORMACIÓN; ARTE COLABORATIVO; TÁCTICAS DISCENTES; PEDAGOGÍA CRÍTICA}

\section{COLLABORATIVE ENGINEERING AND SELF- LEARNING MODES. THE AULABIERTA EXPERIENCE AT THE UNIVERSITY OF GRANADA}

\section{Abstract}

The article focuses on student's working ways within the Aulabierta experience (University of Granada, 2004 to 2012). Aulabierta was an experimental learning community co-designed by students from various disciplines with the help of a large number of collaborators. This connective place followed the inertia of other critical cultural projects that aim to transform and contaminate the watertight categories established since modernity in the institutions of knowledge. Aulabierta tries to institute dissident ways of working from within the academy to advance in the collective production of knowledge, taking up the political character of this as something that is collectively constructed.

Keywords: SELF-EDUCATION; COLLABORATIVE ART; LEARNING TACTICS; CRITICAL PEDAGOGY

\footnotetext{
Collados Alcaide, Antonio. 2020. "Ingeniería colaborativa y modos de autoaprendizaje: La experiencia de Aulabierta en la Universidad de Granada". AusArt 8 (1): 195-219. D0I: 10.1387/ausart.21656
}

\section{AUSART}




\section{INTRODUCCIÓN}

En la última década han emergido un conjunto de iniciativas planteadas por artistas y colectivos de artistas para desarrollar plataformas críticas de autoeducación insertas en instituciones culturales y educativas o al margen de éstas. Algunos analistas han visto en ellas una tendencia "educativa" en las prácticas artísticas - dándose lo que Irit Rogoff denómino como 'educational turn' (2008)-, por la que muchos trabajadores culturales han desarrollado estrategias que se sirven de medios y formatos educativos, incluido el desarrollo de proyectos donde el formato 'escuela o academia' se toma como modelo para generar espacios de experimentación cultural y política. Esta tendencia coincide con ciertos procesos dados dentro de los movimientos de autonomía para impulsar plataformas educativas alternativas, al modo de 'universidades libres' o centros de estudios independientes. En sinergia con ambas trayectorias reconocemos algunos modelos y ejemplos históricos que podríamos resaltar, como la Free International Universtity fundada en Düsseldorf a principios de los años setenta por Joseph Beuys con la ayuda de algunos colaboradores. Unos años antes, también en la Academia de Bellas Artes de Düsseldorf se auto-organizó un grupo, encabezado por el pintor Jörg Immendorff, que tenía como objetivo principal generar un programa de formación crítica paralela a la academia. Denominada como LIDL Academy, tuvo un año escaso de duración hasta que el propio Ministerio de Educación y Cultura se posicionó prohibiendo sus actividades en el año 1969. El Atelier Populaire o el Poster Workshop, en París y Londres respectivamente, fueron escuelas de medios tácticos insertas o vinculadas a las Escuelas de Bellas Artes de estas ciudades, en las que grupos de estudiantes organizaron talleres para desarrollar mecanismos y una gran imaginería visual para acompañar las luchas estudiantiles y obreras de la época.

Los movimientos estudiantiles vinculados a las revueltas sesentayochistas revirtieron su acción también hacía dentro de las instituciones académicas, reclamando cambios en su estructura y organización, para hacer de ellas lugares más democráticos y accesibles. Tampoco quedaron a salvo de la crítica ni los currículums y programas oficiales, ni la manera de entender los criterios pedagógicos por los que se regía la educación superior, lo que provocó, en el caso concreto de los estudios artísticos, fuertes luchas por modificar ciertos paradigmas tradicionales: se cuestionó la división disciplinar de los itinerarios formativos, exigiendo la posibilidad de elección y conformación del curriculum por parte del estudiante; se luchó por potenciar la investigación como elemento orgánico dentro de la enseñanza y práctica del arte y el diseño; y se 
propusieron reformas en el sistema de maestría, como la creación de cuerpos de profesores en plantilla e invitados, con lo que multiplicar las perspectivas y aprendizajes de un estudiantado sujeto entonces a la jerarquía limitante y doctrinal del modelo 'maestro de taller'.

Estos fueron algunos de los cambios propuestos y alcanzados gracias a las movilizaciones de la época, cristalizados por ejemplo en la 'okupación', por parte de estudiantes, del Hornsey College of Art de Londres en 1968. Durante seis semanas se desarrolló una atmósfera creativa, cultural y política, que dio lugar a una estructura de gestión experimental de la institución, basada en metodologías participativas y en el ensayo de nuevos modos de organización horizontal o desjerarquizados con los que revertir la autoridad de los patrones de gobernanza de la institución.

Estos ejemplos forman parte de un proceso social reformista, respecto a las instituciones del saber, que también ha afectado a los centros académicos especializados en la enseñanza y crítica de la producción artística: escuelas y facultades de bellas artes. Podríamos generar una amplia genealogía de intentos, realizados al interior de instituciones educativas artísticas, o centrados en sus marcos estructurales, por generar espacios de experimentación, no sólo técnica o formal, sino también política ${ }^{2}$. Es decir, por vislumbrar y practicar modos de hacer en los que la práctica cultural se realice vinculada a la problematización de las condiciones de producción y distribución de la misma.

Aunque en la mayoría de artículos y obras de análisis y reflexión sobre este tipo de casos escasean las referencias a proyectos de ámbito hispano y más aún español, podríamos construir una rica y diversa genealogía de prácticas que remitieran a este contexto. Las investigadoras Selina Blasco y Lila Insúa (2019), a partir también de su propia experiencia de gestión cultural universitaria, están realizando un productivo esfuerzo en este sentido que los interesados en este ámbito de reflexión y producción cultural debemos agradecer.

En esta misma línea, nos proponemos recuperar la experiencia de Aulabierta en la Universidad de Granada, haciendo especial hincapié en sus modos operacionales en detrimento de presentar los resultados más llamativos como el proyecto de auto-construcción de un aulario dirigido por Recetas Urbanas, el jardín colaborativo, las prácticas tecno-mediales en relación al territorio u otras iniciativas en colaboración con instituciones y artistas como el Centro José Guerrero, Antoni Muntadas o Martha Rosler. Pensamos que en este momento de crisis y en el contexto del número de la revista Ausart sería estimulante 
presentar y dar a conocer parte de 'la materia oscura' del proyecto (Sholette 2011), con el interés y deseo de que esto pudiera generar nuevas prácticas y experiencias similares.

\section{PRESENTACIÓN DEL CASO: AULABIERTA, UNA COMUNIDAD DE AUTOAPRENDIZAJE EN LA UNIVERSIDAD DE GRANADA}

Soy de Granada y soy un instintivo, que no creo en la Universidad tal y conforme está la Universidad. Ella representa la cultura de las letras, cultura en la que yo no creo.

El asceta debe de pensar que no es hombre fuera de la sociedad. El intelectual debe darse cuenta de que le hace falta ser acción y reacción...

José Val del Omar (1992)

En junio de 2004 un pequeño grupo de personas vinculadas de modos diversos a la Facultad de Bellas Artes de Granada (estudiantes y profesores fundamentalmente) comenzaron a hablar de lo que finalmente sería Aulabierta. Los primeros debates surgen como reacción inmediata hacia algunos de los condicionantes propios de la Facultad de Bellas Artes de Granada. La ausencia de lugares para el encuentro, de espacios para la creación complementarios a los estrictamente programados para la docencia reglada, limitaba las posibilidades de que personas inquietas, que entendían su aprendizaje desde una acción más directa y comprometida, pudieran llegar a encontrarse, compartir ideas o plantear proyectos en grupo. El edificio que ocupa actualmente la Facultad de Bellas Artes de Granada fue en su origen un centro de internamiento psiquiátrico ${ }^{3}$, por lo que fue diseñado para el aislamiento y el control, según las utopías psiquiátricas de la época. Esta situación, condicionada por la arquitectura del edificio, servía de metáfora para explicar una problemática mayor, que derivaría en la necesidad de realizar una reflexión de fondo sobre la actualización de las propias Facultades de Bellas Artes, de su conexión con el devenir de los contextos social, político y cultural, también profesional, es decir, de su puesta en realidad. 
Las necesidades que genera la práctica artística actual (diversidad de los modelos de gestión, cambiante territorio de las prácticas artísticas, multiplicación de contextos y ámbitos de intervención, etc.), y las expectativas y capacidad conectiva del estudiantado, superan con mucho lo previsto en los programas de las asignaturas, y subsisten en cierta medida por la generación de nuevos espacios para la autoformación, a través de foros extraacadémicos, e iniciativas individuales o de grupos, que no encuentran un respaldo adecuado a sus exigencias por parte de la institución. En este contexto, parecía necesario idear un lugar permeable, un espacio donde experimentar otros modos relacionales y de trabajo colaborativo entre aquellos agentes que entendieran su labor, dentro del contexto académico, desde una posición proactiva, es decir, asumiendo compromisos y responsabilidades en el diseño y construcción del medio y los métodos en los que se desarrolla el aprendizaje.

Con este fin, se imponía alcanzar otros objetivos. Uno de ellos, fundamental para el caso Aulabierta, era ensayar experiencias en las que ajustar las necesidades y deseos de aprender de los estudiantes, con la creación de herramientas y espacios que pudieran conducir a una implicación real y efectiva de estos en el diseño de sus propios espacios y métodos de enseñanza y aprendizaje.

El deseo que parecía emerger en estos debates se emparentaba con una suerte de crítica a la institución académica y a sus modos y estructuras de funcionamiento. Un asunto que venía labrándose en el campo cultural y pedagógico desde mediados de siglo veinte, impulsado por unos movimientos sociales y culturales críticos que azotaban especialmente a las viejas instituciones modernas: el museo, la escuela, la Universidad o los centros psquiátricos (Foucault 1976), entre otros. En este eje crítico y productivo no podemos obviar, o dejar al margen, el ejemplo que en políticas educativas nos pudieran dar los movimientos de renovación pedagógica, de educación popular o de pedagogía crítica o radical. Principalmente nos referimos a la acción que estos desarrollan al respecto de la desjerarquización de posiciones y saberes, a la deconstrucción de las normas y discursos rígidos e imperantes en las instituciones educativas $\mathrm{o}$ al desbordamiento de sus propios límites y fronteras, tanto conceptuales como físicas, al promover una rearticulación de los espacios de aprendizaje en el ámbito de lo público y en el devenir de la sociedad.

Apelo al derecho a la deconstrucción como derecho incondicional a plantear cuestiones críticas no sólo a la historia del concepto de hombre sino a la historia misma de la noción de crítica, a la 
forma y a la autoridad de la cuestión, a la forma interrogativa del pensamiento. Porque eso implica el derecho de hacerlo afirmativa y performativamente, es decir, produciendo acontecimientos, por ejemplo, escribiendo y dando lugar a obras singulares.

(Derrida 1998)

La crítica, entendida por Derrida como deconstrucción debería -según élhallar en la Universidad su espacio de 'discusión incondicional', un lugar de "resistencia crítica frente a todos los poderes de apropiación dogmáticos e injustos" (Derrida 1998). Pero en este espacio, el discurso crítico debiera también performativizarse (Butler 1995) para producir así acontecimientos en los que pudieran aflorar resistencias que fueran más allá de los límites inherentes a los marcos meramente discursivos.

Este asunto es el que mantiene en tensión la pedagogía crítica, en tanto que entiende que todo discurso debe escenificarse para romper cualquier esencialismo, categoría o ley de significación previa. Aún siendo un discurso que se suponga crítico o transformador, éste impone unas jerarquías y relaciones de poder que, al performativizarse, son renegociadas y resistidas por los sujetos y culturas a los que se dirige. Esto quiere decir que, diseñado un escenario discursivo, los agentes que lo escenifican (profesorado y estudiantado, en el caso concreto que presentamos como caso) irrumpen poniendo en pausa, resistiendo o negociando las múltiples relaciones de poder construidas en él. Es decir, se produce una transformación desde dentro, dando lugar a un nuevo escenario donde el discurso sufre múltiples traducciones, donde la crítica ejerce una labor deconstructiva de las posiciones dadas de antemano, implicando con ello un intercambio no solo de conocimientos y saberes sino de las mismas jerarquías y posicionamientos de los sujetos que forman parte de los mecanismos o estructuras donde se efectúa y produce la crítica.

Esta crítica deconstructiva o 'más que crítica' según Derrida, produce, a través de la performatividad de la misma, una re-territorialización de las condiciones dadas dentro de un sistema, haciendo emerger tácticas que podemos denominar 'oposicionales', es decir, formas complejas, que por su condición difusa e incluso híbrida, las hacen dificíles de ser reconocidas y capturadas por el mismo sistema, ya que surgen contra y dentro de él. En estos casos notamos como se conforman espacios de resistencia, 'intersticios' o espacios 'entremedias' (Bhabha 1994) lugares en continua tensión y ruptura, en los que se da una constante negociación e intercambio entre agentes. Es aquí donde puede darse la construcción de una plataforma de producción de saberes realmente 
colectiva y ecológica, es decir, que amalgame, relacione y rearticule las capacidades y experiencias del conjunto de sujetos e instituciones en interacción dentro de un mismo contexto. Podemos hablar entonces de espacios experimentales o laboratorios transfronterizos de producción de conocimientos, ya que actúan traduciendo, multiplicando y dispersando identidades y posiciones dadas, forzando, a través de actos de resistencia, a romper las fuerzas de control y dominio y a experimentar modos relacionales y operativos transliminales.

\begin{abstract}
El proyecto Aulabierta reconoce este marco conceptual y político, aunque fue su devenir experimental el que construyó su propio posicionamiento ideológico. Es decir, surge de una manera descondicionada, también de un modo un tanto ingenuo, a través del impulso y mezcla de intuiciones, ideas y deseos personales que, a través de diversos medios de articulación, encuentran un ímpetu compartido y se disponen a la tarea de formalizarlo.
\end{abstract}

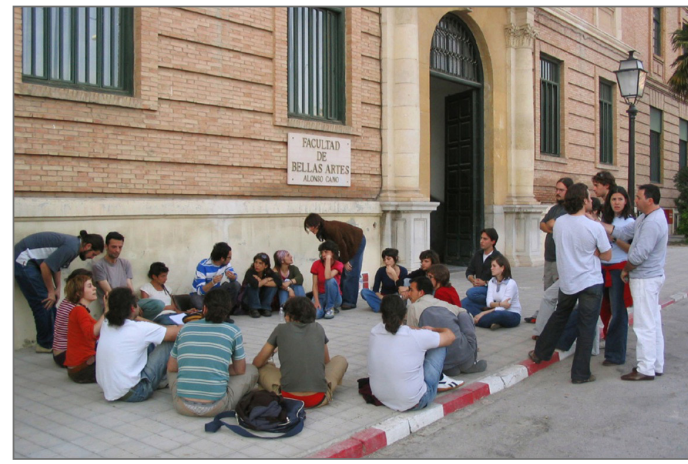

II. 1. Grupo de trabajo formado durante el primer seminario del proyecto Aulabierta en la puerta principal de la Facultad de Bellas Artes de Granada (AAABIERTAprycto, marzo de 2005)

\title{
3. ORIGEN DEL PROYECTO
}

A raíz de los procesos electorales que se dan en la Facultad de Bellas Artes en el año 2004 surgieron algunos debates sobre la necesidad de generar un espacio de trabajo y reflexión crítica en la facultad, más atento a las corrientes actuales del $\mathrm{arte}^{4}$, sin olvidar que su contexto de intervención más próximo, dentro del cual se encontraba, era la Universidad, un centro de investigación y formación, por lo que los debates de carácter epistemológico entraban a la vez de lleno en los objetivos conceptuales y tácticos del proyecto. Sería este un lugar donde organizar actividades y programas complementarios a los planes de estudios que pudieran convertirse en nuevos puntos de encuentro, creación y debate crítico en la Facultad de Bellas Artes. 
Tras el proceso electoral, el profesor Víctor Borrego Nadal, nuevo Vicedecano de Cultura y Alumnos, implicado en estos debates, facilitaría la puesta en marcha de la creación de ese espacio para-académico favoreciendo las condiciones para su realización (capacidad de organización, financiación, difusión, etc.) e implicando a todas las comisiones de Cultura de los distintos Departamentos de la Facultad de Bellas Artes. Borrego Nadal pensaba que las Comisiones de Cultura podían transformar en cierta medida la Facultad porque sin tener que modificar planes de estudio se podía crear una estructura formativa paralela o subliminal a la reglada, mediante la propuesta de organizar ciclos de trabajo temáticos desde el programa cultural del vicedecanato, a los que pudieran sumarse las propias asignaturas, construyendo una red de coordinación entre ellas. La intención final de este impulso era poder hibridar los programas docentes de las asignaturas regladas con las actividades culturales programadas desde el vicedecanato y con experiencias culturales desarrolladas fuera de la Facultad. De esta forma, se podría llegar a crear un sistema permeable en el que se tejiera una red de relaciones más dinámica y flexible, de acuerdo con lo que es común en el sector profesional de las Bellas Artes.

Este plan de actuación tomó el nombre de CICLOTÍMIA y consistía en la creación de una serie de ciclos temáticos, consensuados abiertamente por las comisiones implicadas, sobre los que gravitarían las actividades culturales a celebrar en la Facultad de Bellas Artes a lo largo del curso académico. Con estos ciclos se intentaría incentivar la participación y coordinación de aquellos profesores (y las respectivas asignaturas que impartían) que quisieran sumarse a la red de investigación y formación diseñada desde CICLOTÍMIA, creando con ello una estructura educativa transversal a la reglada.

\section{HERRAMIENTAS Y ESPACIOS: ASOCIACIÓN AAABIERTA, ASAMBLEAS Y ESPACIOS FÍSICO Y DIGITAL}

En paralelo a la creación del programa CICLOTÍMIA, se intentó dar impulso a la creación de asociaciones de estudiantes dentro de la Facultad de Bellas Artes. En el inicio del curso 2004-2005 solamente la Delegación de Estudiantes de la facultad poseía una estructura similar a la de una asociación, pero sin llegar a estar legalmente constituida. 
Con las asociaciones se quería incentivar la creación de equipos a los que pudieran vincularse estudiantes con intereses comunes y voluntad de diseñar y gestionar actividades diversas en función de los fines que se promovieran desde cada asociación. Participaba esta iniciativa del espíritu que movía todo el plan de reestructuración del Vicedecanato de Cultura y Alumnos: implicar a todos los estamentos de la Facultad de Bellas Artes en la creación de un plan de actividades simbiótico al docente, que pudiera enriquecer a éste último fortaleciendo sus puntos débiles, poniéndo énfasis en la participación estudiantil.

Según señalaba el Autoinforme final de evaluación de la titulación de Bellas Artes la participación del estudiantado en los órganos de representación de la Facultad era muy escasa ${ }^{5}$-en algunos casos nula-, lo que también era sintomático de la pasiva relación de los estudiantes con el día a día del Centro.

A través de las asociaciones de estudiantes se intentaría paliar esta situación y favorecer otras dinámicas. Entre ellas: la creación de equipos de trabajo y la autogestión de actividades y proyectos con un carácter más independiente.

En septiembre de 2004 se ponen en marcha dentro de la Facultad de BBAA tres proyectos de asociación: Aulabierta (que cambiaría pronto su denominación por AAABIERTA), Aula Esférica y Aula de Cine.

La manera de entender la naturaleza de la asociación AAABIERTA fue cambiando a lo lardo de los años de funcionamiento del proyecto, en función de los debates que iban sucediéndose entre los miembros más activos, de su propio proceso de expertizaje en formas y modelos de organización y participación, y de las propias necesidades que surgían al paso del proyecto.

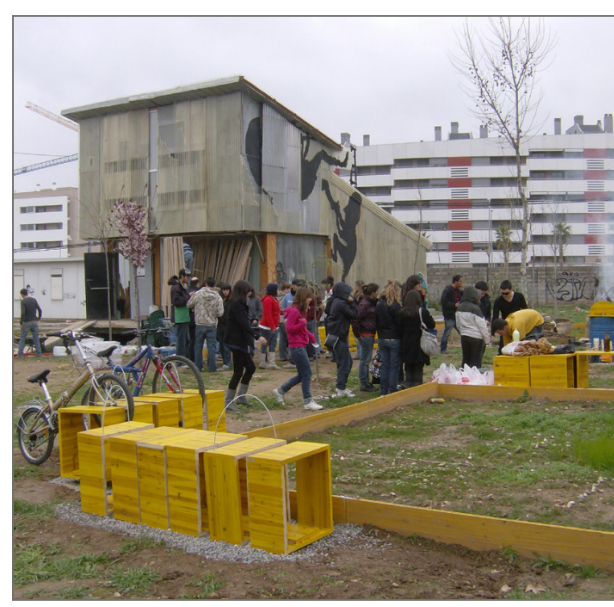

II. 2. Barbacoa en el entorno del aula autoconstruida Dirección del proyecto de autoconstrucción por Santiago Cirugeda (Estudio Recetas Urbanas).

La deriva conceptual y procesual tenida en Aulabierta fue alejando a sus participantes de la idea de comprenderse como colectivo, como grupo homogéneo, encaminándose en cambio a entender la complejidad estructural de Aulabierta como un ensamblaje orgánico de herramientas, puestas a disposi- 
ción de sus usuarios. Un edificio autoconstruido (el símbolo más visible de la iniciativa) [ilustración 2], las distintas webs y listas de correo electrónico, así como la Asociación juvenil y universitaria AAABIERTA, fueron las principales herramientas diseñadas y construidas durante el período de funcionamiento de Aulabierta. Éstas conformaron el organismo que, de distintas formas y con distintos objetivos, articularía la participación de la comunidad de agentes involucrados en la experiencia.

Los modos de organización de los participantes en el proyecto llegaron a ser una consecuencia del uso pactado de estas herramientas, así como, por influencia recíproca, las herramientas fueron adecuándose a los modos de gestión y colaboración que, de manera formal o espontanea, iban emergiendo. Los usos de esta herramientas están sintetizados en el cuadro-diagrama diseñado para formar parte de la hoja comunicación del proyecto [ilustración 3], un material que surge dentro de los continuos procesos internos de redefinición del proyecto, con el objetivo de poder aclarar su estructura y funcionamiento para permitir abrir la participación en él y potenciar su sostenibilidad.

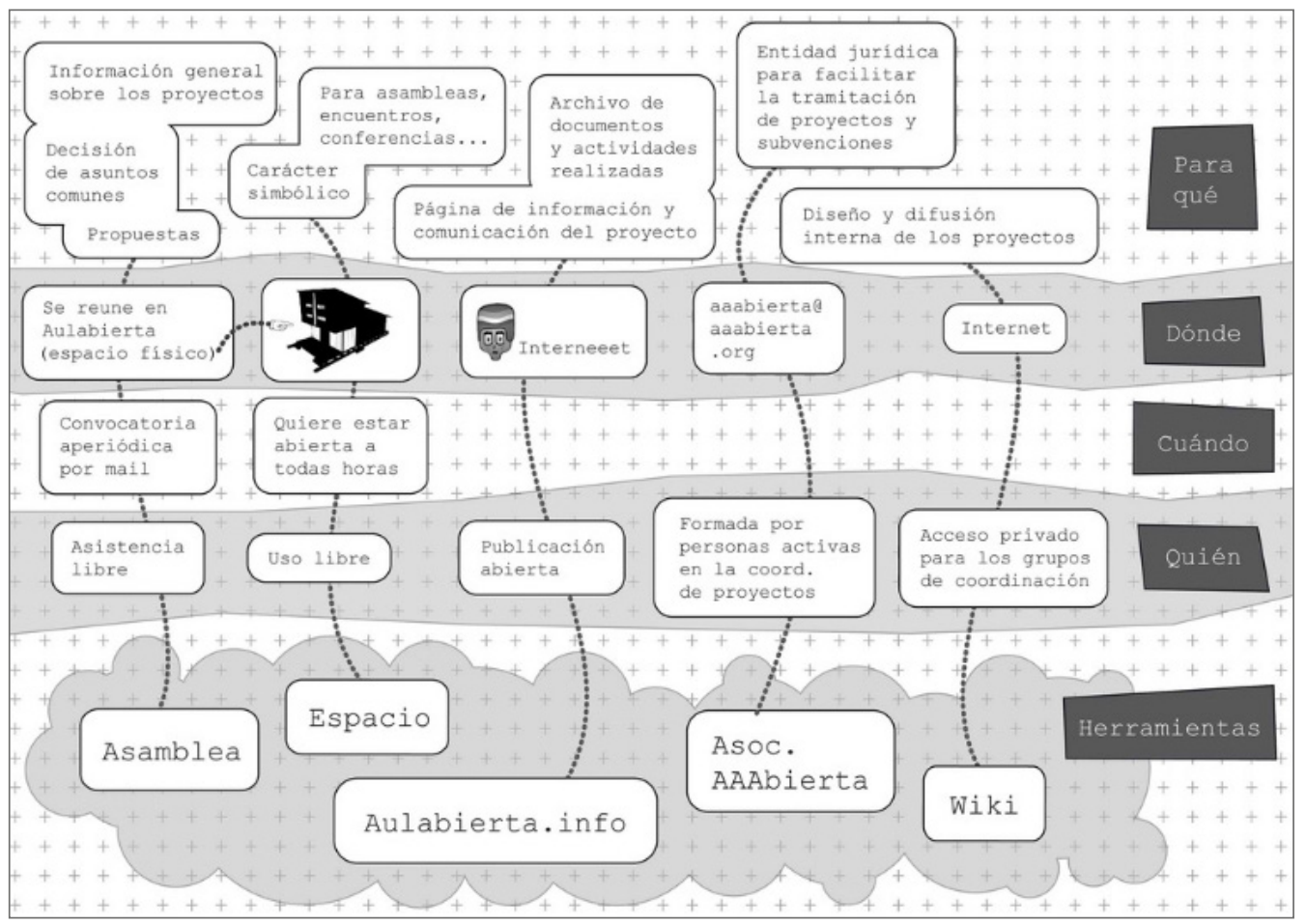

II. 3. Diagrama de herramientas de Aulabierta. Diseño Pablo. P. Becerra y Antonio Collados. 
Fundamentalmente, las herramientas de gestión y organización de Aulabierta se dividían en ${ }^{6}$ :

a. Espacios-herramientas de discusión y gestión: asamblea, listas de correo, wiki.

b. Espacios-herramientas de gestión: asociación juvenil y universitaria AAAbierta (Asociación AulAbierta).

c. Espacios/herramientas de conexión/representación: webs de publicación abierta.

Toda esta serie de elementos funcionaban, dentro del entramado de producción en red de Aulabierta, como una estructura ensamblada de herramientas tecnopolíticas, es decir, como una serie de dispositivos que permitían desarrollar formas de cooperación colectiva, sin demarcar usos concretos. La finalidad de todas estas herramientas, aún descrita y fijada, podía ser alterada, en tanto que se trataba de dispositivos construidos de manera intuitiva y abierta, y que a lo largo del proyecto fueron rearticulándose y modificándose en función de las necesidades y objetivos que podían cumplir en relación al organismo Aulabierta y a la adecuación a su entorno de influencia (la Universidad, el contexto profesional y el local). Por lo tanto, la "caja de herramientas política" de Aulabierta, se construyó y ensayó desde una perspectiva abierta y generativa, como lo hiciera un paquete de software libre, al favorecer un uso no restrictivo y múltiple de sus dispositivos, y al trabajar potenciando la capacidad autónoma de los individuos y grupos de decidir el uso preciso y conveniente de las herramientas, así como la conciencia de estar comprometidos en un proyecto común, en la medida en que la ingeniería social aplicada a las herramientas afectaba y contribuía al común que las comparte.

\section{FUNCIONAMIENTO DE LAS HERRAMIENTAS Y ESPACIOS DE AULABIERTA.}

A partir de la caja de recursos o herramientas descritas anteriormente, el protocolo de funcionamiento para la realización de las actividades y proyectos de Aulabierta [ilustración 4], seguido de manera genérica por los participantes en la experiencia, era el siguiente: 
1. Particulares o grupos presentan y discuten en la asamblea un boceto de la actividad que desean iniciar y producir dentro de los parámetros de Aulabierta. Algunas actividades requieren la formación de un 'grupo de coordinación', conformado en la propia asamblea entre aquellas personas interesadas en involucrarse en la propuesta presentada, otras actividades, no requieren de la construcción de un grupo de gestión, al no ser necesario para su gestión o al ser propuestas realizadas por grupos pre-existentes, los llamados 'grupos de afinidad', formaciones de estructura más estable a las que se deben los proyectos que tuvieron más largo recorrido en Aulabierta.

2. Conformados los grupos de trabajo y gestión, Aulabierta proporciona sus herramientas para facilitar la producción de las actividades y proyectos: su personalidad jurídica (CIF), el registro en el censo de asociaciones regionales y universitarias, lo que posibilita concursar en convocatorias de subvenciones, sus espacios digitales de registro y difusión de actividades. La gestión de proyectos bajo el paraguas de Aulabierta no solo permitía a los grupos contar con la legitimación obtenida gracias a la realización de múltiples actividades, sino que como consecuencia de esto, ponía a disposición de los participantes en el proyecto, la acumulación de un currículum vitae construido de manera colectiva.

Las herramientas digitales, como el wiki, eran utilizadas para dar entrada y registro, de los procesos que afectaban al proyecto, así como de las distintas actividades pasadas y en marcha. Una de las condiciones para la realización de proyectos y actividades dentro del marco de Aulabierta, era abrir una página de información en el wiki sobre cualquier proyecto que se activara, dando cuenta para el conjunto de participantes en Aulabierta de los objetivos y estado permanente de los procesos que se estuvieran llevando a cabo. Este sistema permitía realizar un continuo trasvase de información entre los distintos grupos y personas vinculadas a Aulabierta, permitiendo realizar una formación indirecta mediante la lectura de la información, negociaciones y estrategias relatadas en estas páginas del wiki. Por otra parte, la web de Aulabierta (www.aulabierta.info, actualmente desaparecida), facilitaba un tablón de anuncios público a disposición de los proyectos. A través de sistemas de sindicación y suscripción al boletín de noticias, la difusión externa de las actividades contaba con una herramienta de alcance medio-alto en relación al radio de parti- 
cipación e influencia de las actividades de Aulabierta: eminentemente local y universitario.

3. Una vez registradas las actividades en el wiki, los grupos de coordinación se auto-organizaban para realizar el diseño de la actividad y proyecto y hallar los medios y recursos con los que posibilitar la producción de la propuesta. En ocasiones el grupo requería del apoyo en el diseño de algún o algunos 'colaboradores'. Esta figura fue cubierta tanto por profesorado adscrito a la Universidad de Granada, quienes facilitaban gestiones internas (solicitudes, firmas de aval, permisos, etc.), como por profesionales externos, expertos a los que se recurría en busca de un apoyo en el diseño y dirección de alguna de las líneas de trabajo. Entre los participantes en Aulabierta se construyó la idea de que estos profesionales actuarían como complemento docente, algo así como la figura del 'profesor invitado' habitual en los centros universitarios, con la salvedad de que la propuesta de vinculación era en este caso gestionada y realizada desde Aulabierta, es decir, desde el estudiantado.

4. Conformados los equipos de coordinación (estudiantes, acompañados de colaboradores internos y externos), se llevaba a cabo el diseño y, en caso de que fuera necesario, la tramitación académica de la actividad, ya que muchas de las propuestas desarrolladas desde Aulabierta adoptaron la forma de cursos de formación complementaria oficiales ofertados por la Universidad de Granada. Estos equipos tenían a su cargo la búsqueda de apoyos institucionales externos a la Universidad de Granada, bien para conseguir financiación para las actividades o recursos materiales necesarios para el desarrollo de las mismas.

5. Una vez quedaba resuelta la producción de la actividad o proyecto, a través de los canales de difusión de Aulabierta, se generaban convocatorias abiertas de participación, mediante las cuales se invitaba a estudiantes a inscribirse en la actividad, en ocasiones pagando una pequeña matrícula o de forma gratuita.

6. Una vez resuelta la producción de la actividad y su celebración se generaba habitualmente un acta a modo de memoria de lo sucedido. En el wiki de Aulabierta, se publicaban las actas y memorias de evaluación de las actividades, y en la web pública www.aulabierta.info, 
las hojas de información y memorias audiovisuales de las mismas (imágenes y vídeo resumen). Esto daba lugar a una rearticulación de los saberes producidos, mediante la difusión de contenidos y la multiplicación de los procesos emprendidos en nuevos proyectos, gestionados por los mismos grupos de coordinación o por otros surgidos de los encuentros entre agentes que auspiciaban las actividades desarrolladas. En este sentido, podemos hablar de la construcción de una red de aprendizaje político, en tanto que, no se limitaba a una transmisión meramente de contenidos, sino al desarrollo de estrategias de coaprendizaje táctico, mediante las cuales poder desarrollar modos de apropiación y subversión de los protocolos de diseño y realización de los programas, proyectos y actividades docentes de la Universidad.

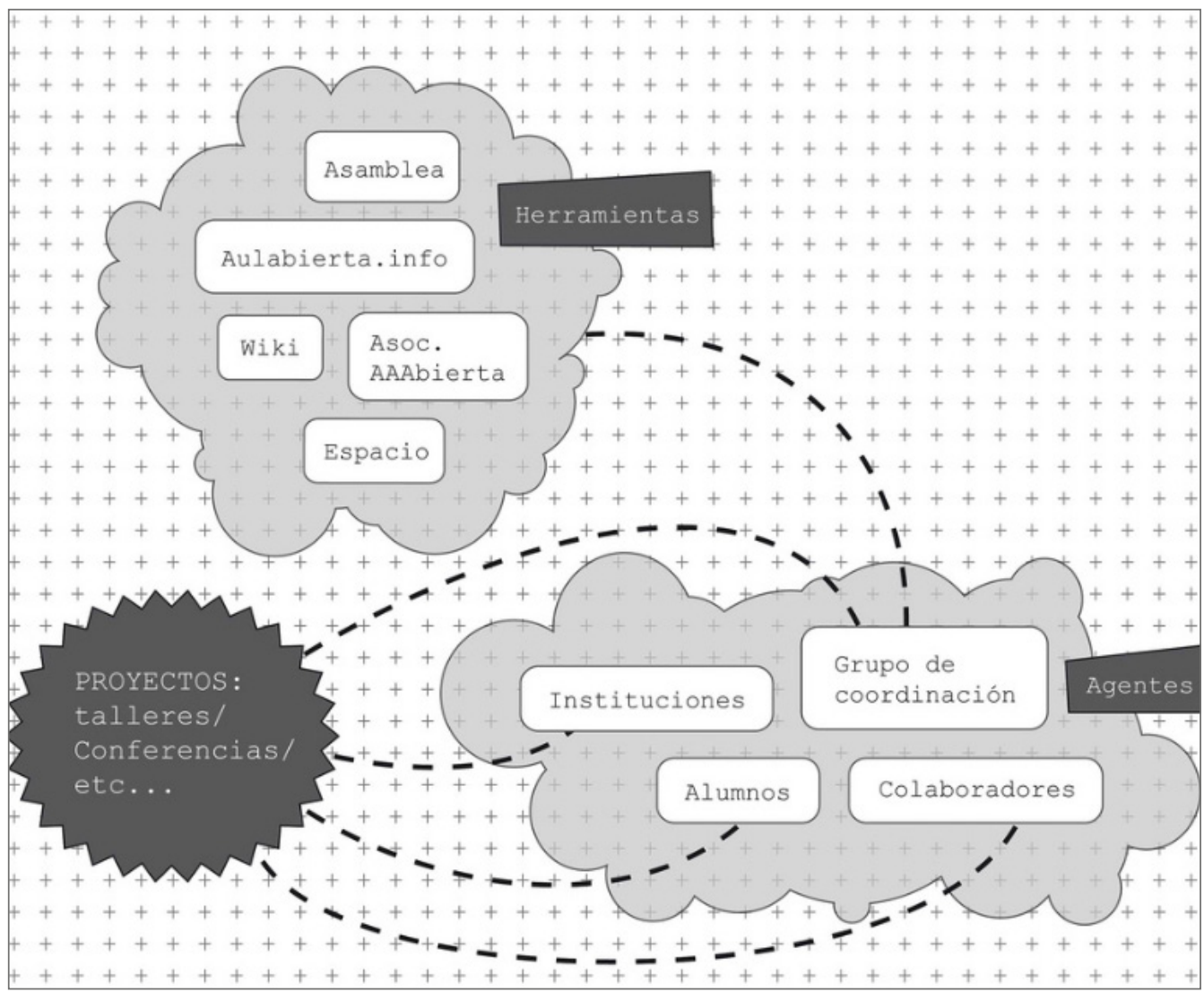

II. 4. Diagrama de protocolos de gestión de actividades en Aulabierta. Diseño Pablo. P. Becerra y Antonio Collados. 
La secuencia de trabajo anterior la entendemos como un ejemplo de pedagogía relacional y política, en tanto que construye una suerte de saberes tácticos en continua rearticulación y reapropiación. En este sentido, la caja de herramientas que componía el organismo Aulabierta debería entenderse como un dispositivo de enunciación múltiple y diversa, en el que el conocimiento se construye social y relacionalmente, a través de las intersecciones que se producen entre los agentes, las herramientas y el medio, sistema o contexto donde intervienen. Así, podemos entender Aulabierta como un organismo de carácter ecológico y crítico, que no niega su contexto de actuación, la Universidad, sino que trata de insertarse en él, reformulando de manera proactiva las fórmulas y medios del sistema donde actúa para trabajarlos subversivamente. En el caso de Aulabierta, ésta, surge dentro de la Universidad, aprende de su medio y trata de deconstruir sus fórmulas de organización y funcionamiento para, con ello, conseguir utilizarlas con otros fines y propósitos. En este sentido, el trabajo de Aulabierta aparece articulado, como ayudaba a comprender el investigador y educador Javier Rodrigo, como un conjunto de acciones de "guerrilla político-educativa [...] mediante las cuales Aulabierta utiliza los códigos del lenguaje oficial, los instrumentaliza y los parasita a través de enunciamientos colectivos" (Rodrigo 2008).

Aulabierta, a partir de complejas negociaciones, intentaba romper las rutinas y ciertos anquilosamientos impuestos por el sistema universitario. Para ello, releía de manera subversiva y proponía, a partir de la acción colectiva, un trabajo de resistencia política que trataría de conocer el sistema para desbloquear o romper las relaciones de poder y jerarquías verticales impuestas.

La práctica política de Aulabierta, es decir, sus formas y modos de actuación suponen, según lo que Eduardo Serrano define como 'tecnología inversa' (2005), un ejercicio de descomposición o deconstrucción de los elementos que articulan la estructura o sistema por el cual se transmite el conocimiento en el ámbito universitario. "Las tecnologías inversas hacen lo contrario que las tecnologías convencionales (o directas): si éstas componen elementos seleccionados de los procedimientos pretecnológicos, la inversión conduce a una descomposición (deconstrucción) y a una restitución de esos fragmentos a un flujo maquínico sin dueños privados: lo común" (Serrano 2006).

Este sistema quedó sistematizado en la gráfica titulada "Sistemas de diseño de programas docentes en la UGR y Aulabierta" [ilustración 5] con el que trataba de ilustrarse la inversión de responsabilidades que tenía el estudiantado 
en Aulabierta, respecto a la Universidad, en la construcción de sus itinerarios de aprendizaje académico.

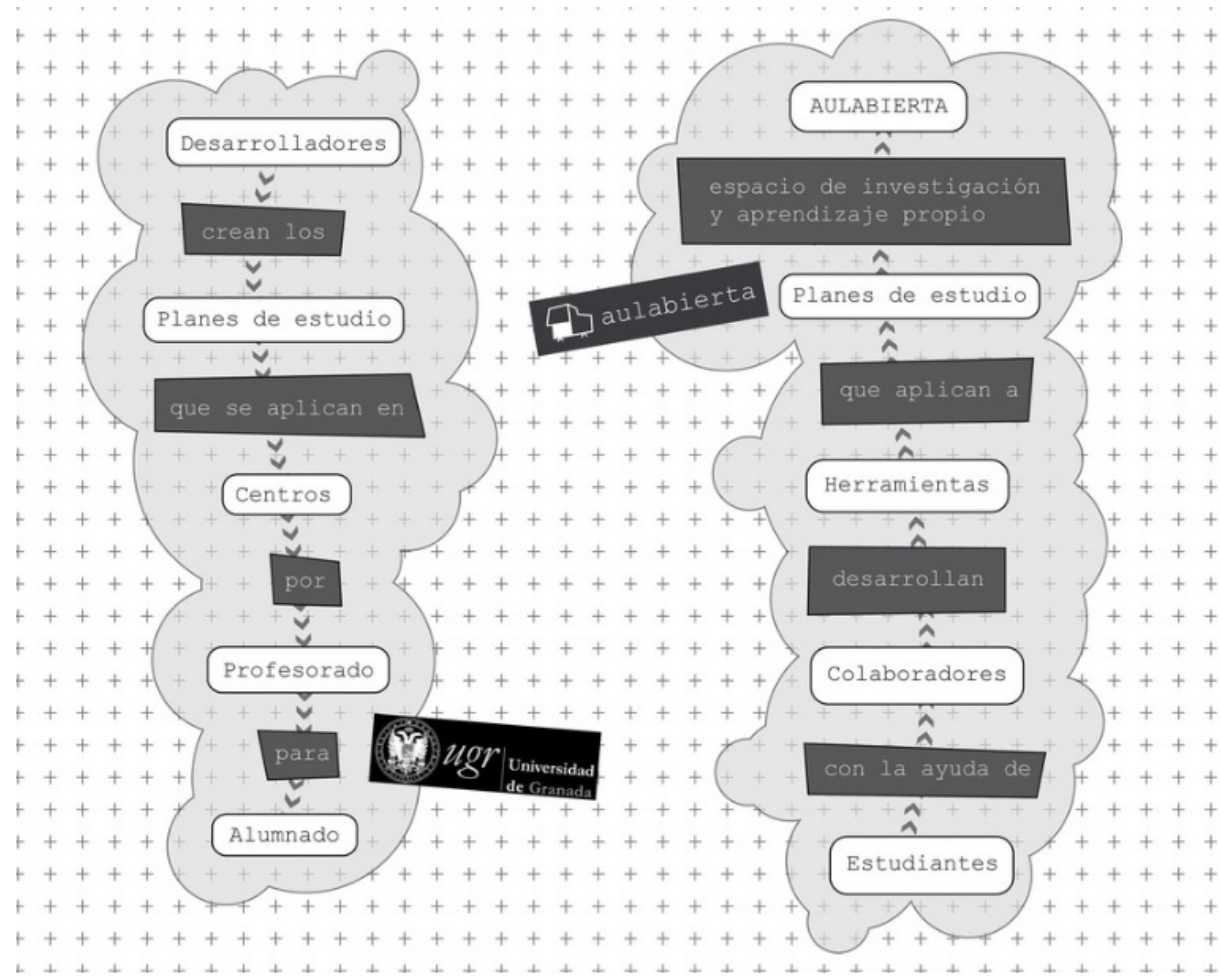

II. 5. Sistemas de diseño de programas docentes en la UGR y Aulabierta. Diseño Pablo. P. Becerra y Antonio Collados.

El sistema da cuenta de la construcción del espacio de aprendizaje colectivo que quería ser Aulabierta, posible mediante la participación activa del estudiantado (acompañado de expertos colaboradores) en el diseño de herramientas y estrategias discentes propias. Una estrategia inversa al sistema universitario habitual, el cual vendría demarcado por una aplicación vertical de planes de estudio desarrollados sin la participación directa de los estudiantes. En este sentido, lo que trataba de evitar el sistema de herramientas y espacios experimentados en Aulabierta era caer en un predeterminismo pedagógico reactivo al académico-formal, pero que en esa reacción, construyera un marco tan cerrado e impositivo como el que cuestiona. Es decir, lo que trataba de borrar Aulabierta, es la diferencia relacional entre los distintos sujetos que participan del espacio académico, para aplanar con ello jerarquías y funciones, hasta conseguir un espacio donde fueran los propios sujetos los que decidieran y 
pudieran construir el modo, los métodos y los conocimientos que deseaban adquirir, a través de procesos de auto y cogestión.

Más claro aún, la metodología inversa de Aulabierta trataba de evitar el generar paquetes o programas educativos ad hoc, específicos o cerrados, que pudieran responder únicamente a una idea o discurso educativo y/o cultural concreto. En cambio, esta metodología enfatizaba la naturaleza relacional del conocimiento, permitiendo que cualquier sujeto pudiera multiplicar su capacidad de acción y participación en el sistema académico, intentando además romper las distancias que demarcan ciertas categorías tradicionales (educador/educando, experto/lego, evaluador/evaluado, etc.), desterritorializándolas o desbordándolas incluso.

En ello vemos emerger un modelo pedagógico de carácter rizomático. Retomando la teoría del conocimiento rizomático de Deleuze y Guattari (1977), el investigador Javier Rodrigo propuso esta forma de entender el trabajo político-educativo de Aulabierta, al notar la querencia y voluntad del proyecto por indagar y construir formas de conocimiento no dicotómicas ni jerárquicas (vertícales y arbóreas), sino tendentes en cambio a la invisibilidad o subliminalidad, a la transversalidad y multiplicidad, a la ruptura y el desequilibrio.

Una pedagogía rizomática sería, según Rodrigo (2008), aquella que "crece $y$ se dispersa transversalmente, de forma colectiva en un comportamiento intersticial. Sus frutos son inesperados, su crecimiento y extensión también. Es por ello que la pedagogía entendida así como elemento político, sólo existe en su múltiple fluir, como continua reemergencia en huecos, estructuras y espacios inesperados, siempre reconectándose, dispersándose, fluyendo y desbordando".

Este carácter intersticial que se entrevé en los modos de hacer políticos de Aulabierta traría de constituirse en una estrategia con la que escapar de las formas de dominación y control que condicionan el orden de lo cotidiano. Con ello, y queda ejemplificado en el marco de intervención del proyecto, Aulabierta no trata de multiplicarse al margen del contexto del que surge, la Universidad, sino que su trabajo se inserta y performatiza en ese ámbito, hasta conseguir dar lugar a un espacio simbólico, pero también de contacto, donde las normas puedan traducirse de manera crítica o subversiva. 


\section{MECANISMOS Y HERRAMIENTAS PARA UNA PRÁCTICA AUTOFORMATIVA EN LA UNIVERSIDAD}

Como hemos intentado explicar con anterioridad, Aulabierta emerge dentro del sistema universitario, no renuncia a trabajar dentro de él, ni trata de mantenerse al margen del conjunto de normas y convenciones que guían la vida universitaria. Trata, sin embargo, de hacer de ello su motivo o razón de trabajo: cómo revertir la normatividad académica, en demasiadas ocasiones rígida e inadecuada al deseo del estudiante, subvirtiendo sus códigos y estructuras funcionales. En este sentido, Aulabierta utiliza los sistemas académicos, los analiza y deconstruye, para reapropiarse de ellos y traducirlos en engranajes que hagan posible la continua circulación de los saberes colectivos que aspira a producir.

Los engranajes que vamos a exponer a continuación, forman parte del sistema o caja de herramientas tecnopolítica que Aulabierta compuso en función de los procesos de gestión táctica ${ }^{8}$ que ha desarrollado. A partir de la reapropiación y uso subversivo de determinados medios y herramientas en funcionamiento en la Universidad, Aulabierta concibió su modo de 'penetrar' o deslizarse dentro de las propias dinámicas de formación universitarias. Es decir, entendemos que los modelos tácticos que siguen, son la metodología empleada por Aulabierta para influir y ejercer una transformación real del contenido y forma de la enseñanza y el aprendizaje dentro del sistema universitario en los años de actividad del proyecto.

\section{A) Créditos de libre configuración}

Las universidades españolas reservaban un porcentaje de créditos, no inferior al $10 \%$ de la carga lectiva global, a la libre configuración curricular$^{9}$. El énfasis táctico se puso entonces en asumir la responsabilidad en Aulabierta de proponer y diseñar programas y proyectos formativos propios. Para ello, se necesitaban fórmulas posibles para convertir los deseos de aprendizaje, las materias y contenidos que querían introducirse en el plan de estudios, en actividades que pudieran ser reconocidas académicamente. No se trataba de generar un sistema de cursos o actividades complementarias a las docentes, sino de intentar encontrar los mecanismos por los que las propuestas surgidas del entorno de Aulabierta pudieran permear el propio curriculum académico. Con ello 
trataría de probarse la posibilidad de que el estudiantado pudiera diseñar parte del plan docente de su titulación.

\section{B) InCLUSión DE ACTIVIdAdes Y PROYECTOS DENTRO DEL PLAN DOCENTE \\ DE ALGUNAS ASIGNATURAS}

La segunda vía empleada para conseguir intervenir de alguna forma en el curriculum académico fue la alianza establecida con algunos profesores para diseñar conjuntamente actividades e introducirlas dentro del marco docente de sus asignaturas.

\section{C) Diseño de proyectos de innovación docente}

En última instancia, algunos de los grupos de afinidad o de coordinación de Aulabierta utilizaron tácticas más complejas a la hora de poder generar procesos de autoformación dentro de los marcos de reconocimiento académico. Se inició una indagación y análisis de los protocolos universitarios con el objetivo de entrever, en sintonía con la metodología inversa de Aulabierta, las posibilidades de hacer un uso subversivo de ellos. En este proceso, emergen las convocatorias de 'proyectos de innovación docente' de la Universidad de Granada. Se trata de unos programas de ayudas cuyo objetivo principal es incentivar la mejora de la docencia universitaria para "conseguir los niveles de calidad y excelencia necesarios para una adecuada formación y capacitación de los estudiantes [...] Con la innovación docente se pretende una docencia universitaria de calidad, donde la comunicación creativa y retadora del profesorado con sus estudiantes sea el eje central del proceso docente"10.

Si interpretamos estos propósitos de una forma literal, comprendemos que la tarea desenvuelta en Aulabierta no deja de poner en el centro del espacio de enseñanza y aprendizaje universitario la necesidad de ejercitar políticas colaborativas entre los distintos agentes que forman la comunidad universitaria, para con ello hacer más compleja y rica, y quizás actualizar y reconectar el ámbito académico con movimientos emergentes, con procesos culturales y tecnológicos veloces y líquidos, con saberes que escapan o acontecen más allá de los flancos de la Universidad. 
Este panorama impulsó una táctica complementaria a las anteriores para desarrollar nuevos programas o para calzar existentes bajo el paraguas de la innovación docente. Se trataba de cohesionar a un grupo de profesores, de una o varias titulaciones, para trabajar de manera coordinada en el desarrollo de programas complejos. Esos programas eran diseñados por estudiantes a partir de la creación de grupos de coordinación. Una vez quedaba descrito los ejes conceptuales y prácticos principales, se estudiaba dentro del profesorado universitario quienes podrían estar interesados en desarrollar e implementar la propuesta dentro de su espacio docente. Conformado un grupo se redactaba la propuesta definitiva y se adjuntaba el aval de cada profesor. Si la propuesta obtenía la aceptación del Programa de Innovación Docente, se avanzaba en la articulación del grupo y en la asignación de tareas. Los grupos de coordinación, formados por los estudiantes que avanzaban la propuesta, ejercían la gestión interna del proyecto y la coordinación entre docentes. Éstos, adecuaban y desarrollaban los contenidos y objetivos propuestos en el plan de innovación redactado en sus propias asignaturas, dándose el caso de que estudiantes que habían participado en la redacción de un Proyecto de Innovación Docente participaban como alumnos de su propio plan.

Este es el caso del programa Aulagarden [ilustración 6], una experiencia educativa que tenía como objetivo abrir un proceso de experimentación transdisciplinar para crear un espacio verde en el entorno de la sede física de Aulabierta, un proyecto de paisajismo colaborativo, que pudiera devenir en la creación de un protocolo implementable en otros casos y contextos. La vinculación de asignaturas de diversas áreas de conocimientos (Bellas Artes, Arquitectura, Sociología, Botánica), con sus respectivos profesores y estudiantes, la implicación de Departamentos, centros y oficinas de servicios de la Universidad de Granada, la implicación de profesionales e instituciones externas, hacen de esta fórmula el método quizás más completo y complejo de los experimentados en Aulabierta.

\section{D) Actividades complementarias ajenas al currículo acAdémico}

Por último, debemos apuntar que un buen número de actividades y proyectos desarrollados dentro del engranaje de Aulabierta, lo hicieron al margen del sistema de penetración en el currículo académico que hemos detallado anteriormente. Cursos, talleres, encuentros, etc. 
que se programaron desde la voluntad y el placer por abrir espacios de aprendizaje 'otros', es decir, fuera de los itinerarios académicos, o mejor, del sistema de normas y condiciones que los articulan.

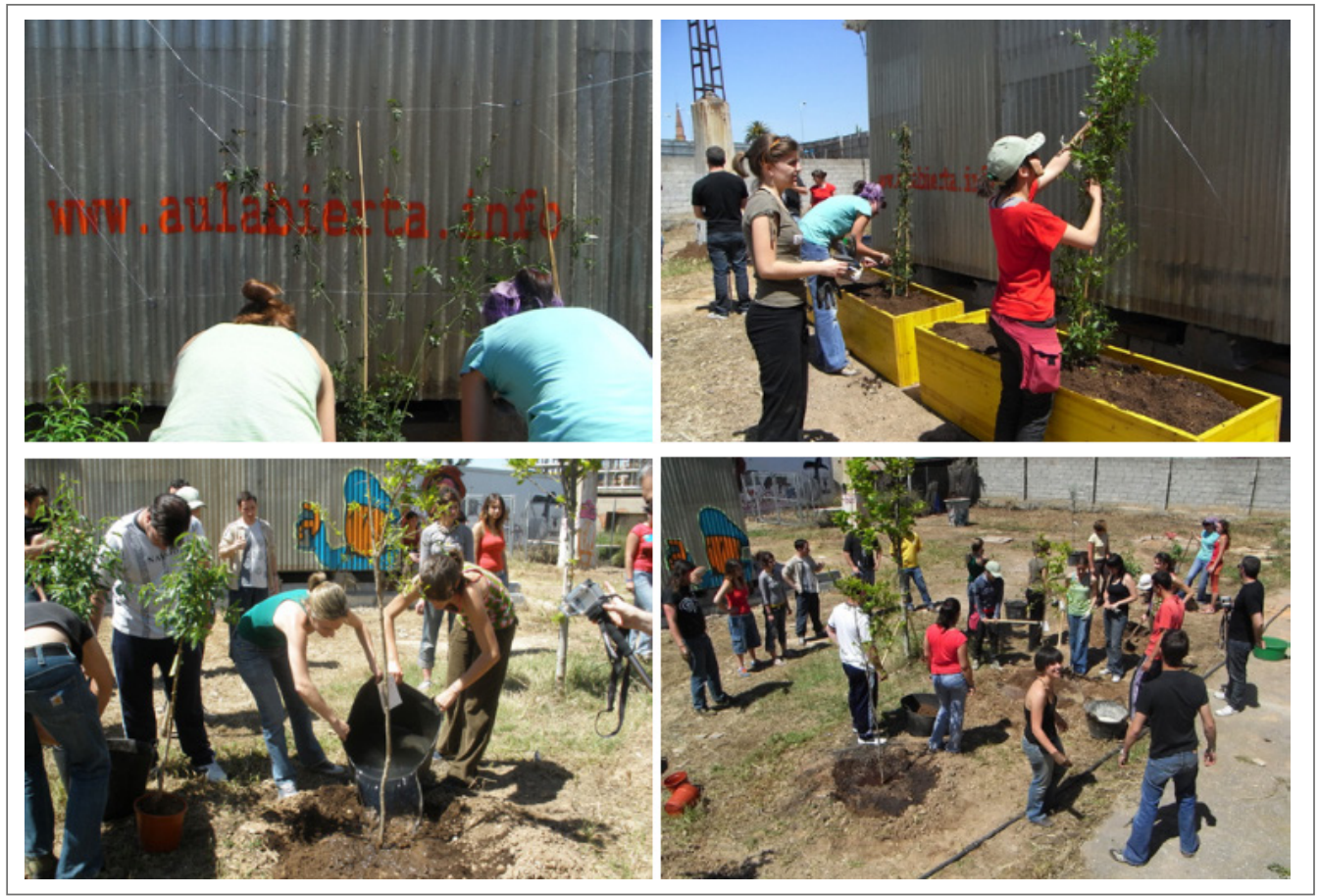

II. 6. Mosaico de imágenes de la práctica de plantación correspondiente a la segunda fase del proyecto de innovación docente Aulagarden. Abril de 2008.

En estas tácticas y modos de hacer se concreta el trabajo de transgresión colectiva que efectúa Aulabierta de las formas, discursos y sistemas de la Universidad. Se comprende en ellas un afán por desentramar las tecnologías universitarias, es decir, por abrir y explicar la manera en que se emplean unos medios concretos para llevar a cabo un efecto: un modo de aprendizaje. Las prácticas anteriores son, como decíamos, el resultado de procesos de ingeniería inversa, de esfuerzos por comprender y difundir fórmulas de acción no reveladas pero posibles, con las que potenciar el deseo de aprendizaje, de una comunidad, la de estudiantes, incentivada en Aulabierta a tomar las riendas del diseño y construcción de su propia práctica discente. 


\section{CONCLUSIONES}

Hoy día, frente al continuo asedio de nuestras mentes y vidas, de nuestras capacidades y potencialidades, se impone, parafraseando a Marina Garcés (2010), crear nuevos espacios para el aprendizaje y la experimentación cultural, social y política. Lugares comunes que puedan construir resistencias frente a la presión mercantil, la competitividad, la cultura del ocio, la hiperregulación institucional, la caducidad de las ideas, y la precariedad de las relaciones.

El pedagogo crítico Henry A. Giroux (1997) defiende la necesidad de entablar procesos de deconstrucción de los marcos monolíticos y de poder en los que se construyen las representaciones y discursos culturales dominantes. Este proceso, señala Giroux, pasa en cierta medida por criticar, perturbar y transformar las políticas culturales universalistas y homogéneas mediante posturas en resistencia. Es decir, debemos entender la manera por la que el poder privilegia e impone determinados discursos, y cómo relega otros a los márgenes, oscureciendo la diversidad, la multiplicidad y la diferencia. Mediante esta comprensión, surge siempre el desafío por emprender nuevas estrategias culturales, educativas y sociales que generen rupturas y discontinuidades en las formas públicas y relaciones sociales estructuradas desde la norma y la dominación. A partir de ahí sería necesario establecer políticas basadas en el reconocimiento de la diferencia, en la oposición y el diálogo, esto es, en la generación de procesos, experiencias, también prácticas instituyentes, que reconozcan estar en una posición relativa, provisional, variable, experimental, movediza, cambiante. Desde aquí surge el llamamiento que hace Giroux a "educadores y trabajadores culturales para que se conviertan en cruzadores de fronteras, comprometidos en un esfuerzo por crear esferas públicas alternativas" (1997, 36), es decir, agentes que socaven las fronteras existentes, forjadas desde la dominación, para cuestionar cómo éstas han sido heredadas y como enmarcan nuestros discursos y relaciones sociales.

El caso de Aulabierta nos ayuda a entrever posibles recursos y modelos instituyentes, de trabajo colaborativo y de transformación de los modos discentes en el ámbito universitario que, sin obviar sus puntos ciegos y problemáticas, nos ofrece otras posibles formas de participar en la construcción de los espacios de producción de conocimiento y aprendizaje en un momento de crisis como el actual. Quizás los acontecimientos presentes provocados por la crisis de la Covid-19, que viene a iluminar de nuevo los efectos de la crisis del 2008, podrían aportar elementos productivos o abrir nuevas fricciones e intersticios en los que encontrar una oportunidad y espacio para poder pensar la Universi- 
dad en su conjunto. Esta nueva crisis nos está mostrando otras formas de gestión y trabajo que están desde ya transformando las dinámicas académicas.

De esta coyuntura contextual emergerán nuevas preguntas, nuevos medios, nuevos procesos, que si bien, encuentran reflejo en acontecimientos y experiencias pasadas, plantearan retos que también serán nuevos. No quedará otra opción que permanecer atentos y seguir participando y aprendiendo de los nuevos espacios de formulación política y cultural que de manera continua irán surgiendo. De cómo incorporar nuestras mentes y cuerpos, de qué tácticas y modos de hacer poder vincular o extraer en este tiempo, y de cómo hacerlo, puede que sean estas preguntas básicas, pero en su simplicidad recae también la gravedad de este momento. Creemos que el ejemplo de Aulabierta puede aportar elementos singulares y significativos con los que iniciar la tarea de pensar, experimentar y escribir respuestas para las preguntas anteriores y de imaginar nuevas formas de estar en el ámbito académico.

\section{Referencias bibliográficas}

Beuys, Joseph. 2008. Joseph Beuys: Düsseldorf. Stadtmuseum Düsseldorf, 29 Sept.-30 Dec. 2007; text(s) by Jochen Chiout, Susanne Anna, Friederike Schuler, Ralf Zimmer-Hegmann \& Antje Krause-Wahl; Anna, Susanne, ed. Berlin: Hatje Cantz

Bhabha, Homi K. (1994) 2002. El lugar de la cultura. Traducción, César Aira. Buenos Aires: Manantial

Bishop, Claire. 2012. Artificial hells: Participatory art and the politics of spectatorship. London: Verso

Blasco Castiñeyra, Selina \& Lila Insúa Lintridis, eds. 2019. Exterioridades críticas: Comunidades de aprendizaje universitarias en arte y arquitectura y su incorporación a los relatos de la modernidad y del presente. Madrid: Brumaria

— \& Lila Insúa Lintridis. 2018. "Comunidades artísticas universitarias: Respuestas parciales desde exterioridades críticas". [i2] Investigación e Innovación en Arquitectura y Territorio 6(2). https://i2.ua.es/issue/view/774

Butler, Judith. 1998. "Soberanía y actos de habla performativos". Traducción de Ana Romero. Acción Paralela 4. http://www.accpar.org/numero4/butler.htm

Campos Fernández, José Daniel \& Pablo Pérez Becerra. 2009. "Aulabierta: Tácticas discentes". En Accions reversibles: Art, educació, territori [Acciones reversibles: Arte, educación, territorio], exposició comissariada per Ramon Parramon. Vic: ACVIC

Certau, Michel de. (1990) 2000. La invención de lo cotidiano. Volumen I. Artes de hacer. Nueva ed., establecida y presentada por Luce Giard; traducción de Alejandro Pescador. Ciudad de México: Universidad Iberoamericana

Deleuze, Gilles \& Félix Guattari. 1977. Rizoma: (Introducción). Traducción de José Vázquez Pérez \& Umbelina Larraceleta. Valencia: Pretextos 
Derrida, Jacques. (1998) 2001. La universidad sin condición. Traducción de Cristina de Peretti \& Paco Vidarte. Madrid: Trotta. Conf. orig. Univ. Stanford CA, abril 1998

Foucault, Michel. 1976. Vigilar y castigar: Nacimiento de la prisión. Traducción de Aurelio Garzón del Camino. Ciudad de México: Siglo XXI

Garcés Mascareñas, Marina. 2010. "Dar que pensar: Sobre la necesidad política de nuevos espacios de aprendizaje". "El combate del pensamiento", número monográfico, Espai en Blanc 7-8. http://www.espaienblanc.net/IMG/pdf/Dar_que_pensar.pdf

Giroux, Henry. A. 1997. Cruzando límites: Trabajadores culturales y políticas educativas. Traducción de José Pedro Tosaus Abadía. Barcelona: Paidós

Greenwald, Dara. 2010. "School as Art”. Justseeds, 25 feb. https://justseeds.org/school-as-art/

Hargrave, Katie. 2009. "Paulo Freire and educational models as art practice". Blog personal de la autora, 2 abril. http://blog.katiehargrave.us/2009/04/paulo-freire-and-educational-models-as.html

Hernández Calvo, Max. 2011. "La escuela como práctica artística: crítica, recreo y revolución”. Minorliterature (blog), 23 marzo. http://minorliterature.wordpress.com/2011/03/23/la-escuela-como-practica-critica-entre-la-revolucion-y-el-recreo/

Hollert, Tom. 2009. "Art in the knowledge-based polis". E-Flux 3. http://www.e-flux.com/journal/ art-in-the-knowledge-based-polis/

Hornsey College of Art (London). 1969. The Hornsey affair: Students and staff of Hornsey College of Art. London: Penguin

Rodrigo Montero, Javier. 2008. "Aulabierta: Un modelo colectivo de pedagogía rizomática”. Pedagogia: Arte/Diseño-repositorio de referencias enseñanza-aprendizaje. https://eacvvcae.wordpress.com/p-t/rodrigo-javier/aulabierta-un-modelo-colectivo-de-pedagogia-rizomatica/

Rogoff, Irit. 2008. "Turning”. E-Flux 0. https://www.e-flux.com/journal/00/68470/turning/

Serrano Jerez, Eduardo. 2005. "Arte y tecnología inversa”. Vídeo de Dailymotion, conferencia del seminario-taller AAAbierta Prycto. 30-3-05. http://www.dailymotion.com/video/x3qgzi eduardo-serrano-arte-y-tecnologia-i_school

— . 2006. "Carta a los hackitectos". CityWiki.ugr.es. http://citywiki.ugr.es/wiki/Carta_a_los_ hackitectos

Sholette, Gregory. 2011. Dark matter: Art and politics in de age of enterprise culture. London: Pluto

Thorne, Sam. 2017. School: A recent history of self-organized art education. Berlin: Sternberg

Universidad de Granada. 2005. "Informe final de evaluación de la titulación de Bellas Artes”. Vicerrectorado de Planificación, Calidad y Evaluación Docente

Val del Omar, María José. 1992. "Sentimiento de la pedagogía kinestésica (Sedimento emocional de mis experiencias)”. En Val del Omar sin fin, Gonzalo Sáenz de Buruaga \& María José Val del Omar, eds., 57-60. Granada: Diputación de Granada

Vidokle, Anton. 2007. "Exhibition as school in a divided city: An incomplete chronology of experimental art schools". En Taking the matter into common hands: On contemporany art and collaborative practices, Johanna Billing et al., eds. London: Black Dog 


\section{Notas}

${ }^{1}$ Algunas referencias críticas sobre este asunto: (Hernández 2011), (Hargrave 2009), y (Hustseeds 2010).

${ }^{2}$ Esfuerzo por otra parte iniciado por Anton Vidokle para su contribución al texto comisarial de Manifesta 6 (Vidokle 2007, 93-6). Un excepcional estudio ha sido publicado recientemente por el investigador Sam Thorne (2017) en el que genera una genealogía y constelación de prácticas que se mueven entre la institución de espacios alternativos entre el arte y educación: School: A recent history of self-organized art education.

3 "El espacio donde está ubicado la Facultad de Bellas Artes se adecuó de manera provisional con materiales precarios y adaptando un antiguo hospital psiquiátrico. La estructura del edificio es de tipo psiquiátrico-carcelario con enormes corredores que desperdician muchos metros cuadrados y que en ningún modo propician la conexión de los conocimientos sino que todo lo contrario, los aíslan y disgregan" (Universidad de Granada 2005, 36).

${ }^{4}$ El Autoinforme final de evaluación de la titulación de Bellas Artes señala en la misma dirección: "Urge adecuar la enseñanza de las artes al momento actual. Es imposible descuidar las nuevas manifestaciones artísticas y la nueva dimensión con que la sociedad valora prácticas artísticas con tanto peso histórico como la pintura y la escultura, no cabe duda que el lenguaje y los contenidos han dado al traste a lo meramente visual para adentrarse en terrenos más amplios que contienen parcelas incisivas del entorno real. Esta situación debe reflejarse en el día a día de la enseñanza" (Univ. Granada 2005, 33).

5 "Habría que destacar, como punto preocupante, la baja participación de los alumnos en los distintos órganos colegiados. El divorcio del colectivo del alumnado con la marcha organizativa del centro no contribuye al buen desarrollo de los planes de mejora que se establezcan" (Univ. Granada 2005, 34).

${ }^{6}$ Esta división fue propuesta y fijada en el artículo que Pablo P. Becerra y Jose Daniel Campos Fernández escribieron para su participación en el encuentro "Acciones reversibles. Seminario sobre arte, educación y territorio" (2009, 316-22). En él puede leerse una explicación extendida de las herramientas de gestión y organización de Aulabierta.

${ }^{7}$ Estos 'grupos de afinidad' se formaron generalmente a través del encuentro en Aulabierta. Algunos de estos equipos continuaron su trabajo, una vez egresados sus miembros de la Universidad, a través de la creación de empresas u otras estructuras profesionales como fueron el estudio de arquitectos Mytaki (http://mytaki.es/) y el colectivo de productores culturales FAAQ (http://faaq.info/).

${ }^{8}$ Frente a la 'estrategia', en la que se establecen relaciones de amigo-enemigo y jerarquís y territorios marcados, Michel de Certau (1990) reconoce en las 'tácticas' unos usos del espacio y tiempo ni tan jerarquizados ni tan predeterminados. Las 'tácticas' son formas de resistencia y subversión de códigos a partir de una consideración de los usos del tiempo y el espacio de una manera más impredecible e inmediata.

${ }^{9}$ Información sobre Libre configuración curricular recogida en el Art. 7.2.b del Real Decreto 1497/1987, de 27 de noviembre, modificado por el RD 1267/1994.

${ }^{10}$ Texto de la Convocatoria de proyectos de innovación docente para el curso académico 2008/2009. http://www.ugr.es/ secredei/bougr/acuerdos/proyectos_innovacion230608.pdf 